\title{
COVID-19-Associated \\ Neurological Manifestations
}

Layla Southcombe

Editorial Assistant, EMJ

Citation: EMJ Neurol. 2020;8[1]:19-21.

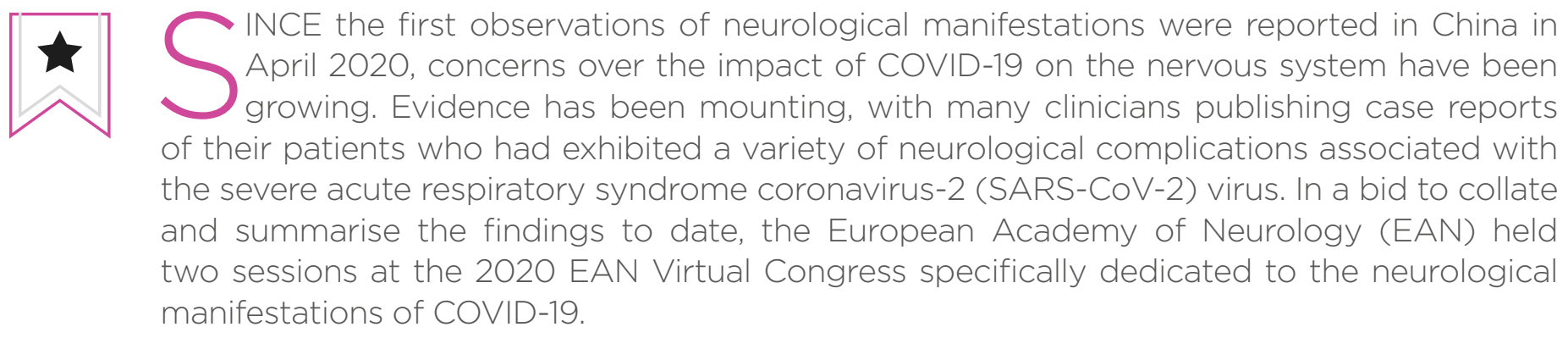

\section{SYMPTOMS AND PREVALENCE}

Preliminary data from published papers suggest that the presence of neurological manifestations in hospitalised patients with COVID-19 was relatively frequent, with a reported prevalence of $36.4 \%$, which increased in those with severe COVID-19." In the first session titled "EAN/ AAN Session on COVID-19," Prof Elena Moro from Grenoble Alpes University, Grenoble, France, presented the preliminary results from registries and surveys recording the neurological implications associated with COVID-19 across Europe. Of note, the most frequent neurological manifestations are headache, anosmia (loss of the sense of smell)/ageusia (loss of taste function), cerebrovascular events, and encephalopathies, with many others having been reported.

\section{DIRECT CENTRAL NERVOUS SYSTEM INFECTION}

In the second COVID-19-dedicated session "The Neurological Implications of COVID-19," Prof Renaud Du Pasquier from Lausanne University Hospital, Lausanne, Switzerland, explained that
SARS-CoV-2 binds to the receptor angiotensinconverting enzyme-2 (ACE-2) via its spike protein. Prof Du Pasquier later provided mRNAbased evidence that ACE-2 is present in the brain, suggesting that there is a possibility that cells of the central nervous system (CNS) can be infected with SARS-CoV-2. ${ }^{2}$

The olfactory nerves have been hypothesised to be one potential SARS-CoV-2 entry route into the CNS. Prof Du Pasquier commented that: "The high number of COVID-19 patients with anosmia clearly suggests that this virus has a tropism for this part of the brain." He added that there would be more evidence of CNS infection in patients with SARS-CoV-2 if this were the predominant mechanism, acknowledging that there have been limited reports of cerebrospinal fluid being positive for SARS-CoV-2 in patients with COVID19-associated neurological manifestations.

The consequences of such direct SARS-CoV-2 CNS infection have emerged as encephalitis and meningoencephalitis, and potentially myelitis. Prof Du Pasquier exemplified the possibility of psychiatric symptoms in patients positive for SARS-CoV-2 in a case of a 64-year-old woman who presented to his clinic with acute psychotic 
symptoms despite having no psychiatric history. After a full work-up and positive nasopharyngeal swab for SARS-CoV-2, the manifestation was diagnosed as meningoencephalitis, most likely related to COVID-19.

\section{INDIRECT INFECTIOUS EFFECTS ON THE CENTRAL NERVOUS SYSTEM}

Hypercoagulable states in patients with COVID-19 have been reported at alarming frequencies, with many resulting in the occurrence of stroke, even in those without any cerebrovascular risk factors. This hypercoagulability is thought to be a result of the activation of inflammatory and thrombotic pathways from SARS-CoV-2 interaction with ACE-2 on endothelial cells, evidenced by pathology studies shown by Prof Moro.

In an autopsy study of 12 patients who died from COVID-19, Prof Du Pasquier highlighted that more than seven had undiagnosed deep vein thrombosis and that four died as a direct result of pulmonary embolism. ${ }^{3}$ There is clearly a trend towards a procoaguable state, he commented, which puts patients at a high risk of stroke. Prof Kenneth Tyler from the University of Colorado School of Medicine, Denver, Colorado, USA, a speaker in the first session, presented studies that reported a delay in cerebrovascular event occurrence, which happened 8-24 days after the onset of COVID-19 symptoms. ${ }^{4}$

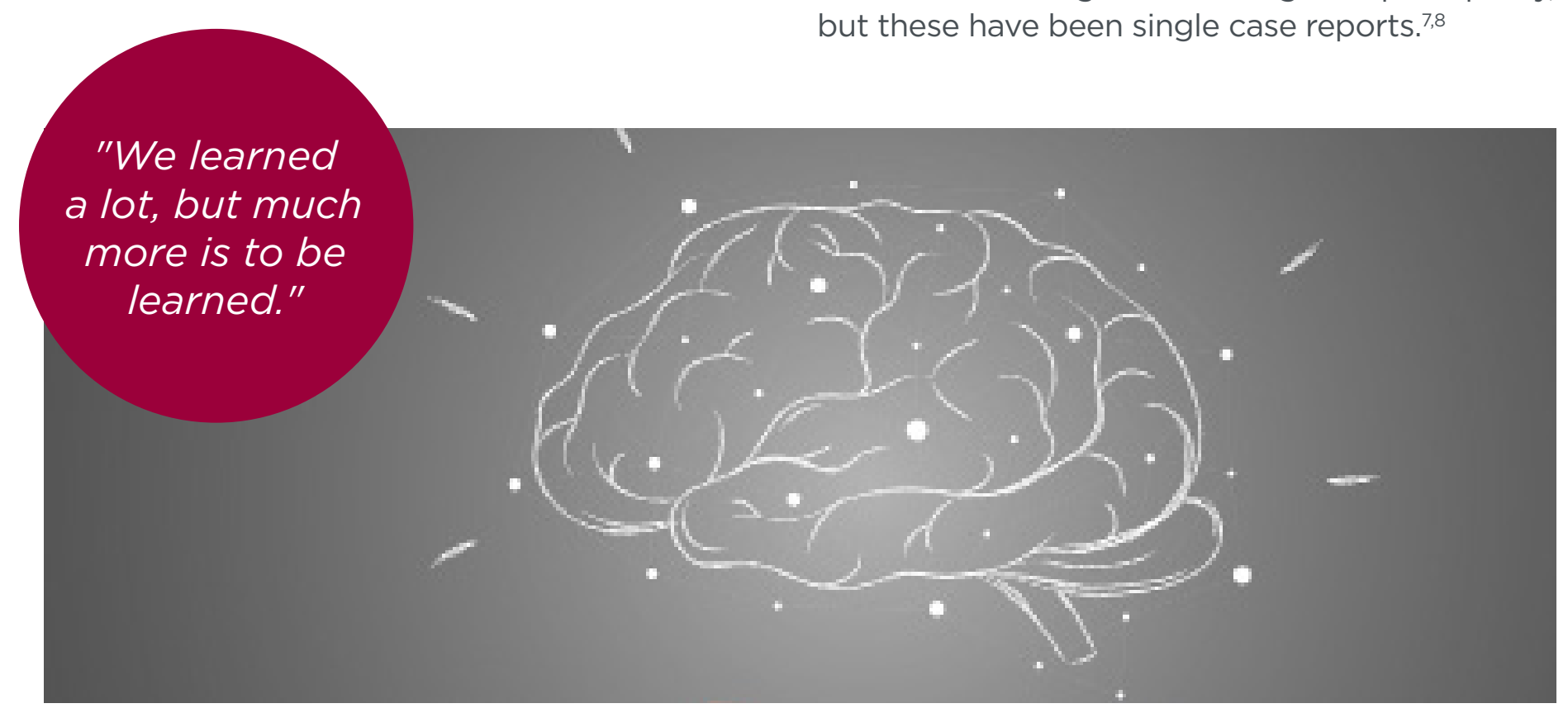

In mild COVID-19, the virus is rapidly cleared by the immune response and the infection is contained; however, in severe disease the viral clearance is suboptimal and a proinflammatory response takes place. Instead of a direct infectious effect of the virus, this SARS-CoV2-triggered mass release of proinflammatory cytokines such as IL-6, IL- $\beta$, and TNFa, the socalled cytokine storm, is another possible cause of the meningoencephalitis seen in patients with COVID-19. A Kawasaki-like disease associated with COVID-19 has also been reported. Prof Tyler presented a published case series in which two patients also had neurological symptoms, mostly in the form of meningeal symptoms. ${ }^{5}$ Prof Tyler added: "This raises the possibility that there are going to be new syndromes emerging with COVID-19 that we haven't yet covered, potentially including vasculitic ones."

\section{POST-INFECTIOUS NERVOUS SYSTEM COMPLICATIONS}

Guillain-Barré syndrome (GBS) is an immunemediated neurological complication that can occur following infection. Numerous small case series on GBS associated with COVID-19 have already been published, but Prof Tyler highlighted one article specifically, in which five patients developed a clinical syndrome compatible with GBS 5-10 days after COVID-19 symptom onset. 6 He added that further postinfectious complications have been reported, such as acute disseminated encephalomyelitis and acute haemorrhagic necrotising encephalopathy, but these have been single case reports. ${ }^{7,8}$ 
indirect infectious manifestations, whether these complications can be treated in similar fashions to other neuroinvasive and neurotrophic infections, and if there is brainstem involvement in the worsening of acute respiratory distress syndrome.

"This raises the possibility that there are going to be new syndromes emerging with COVID-19 that we haven't yet covered, potentially including vasculitic ones."

\section{KNOW THE SYMPTOMS}

Prof Du Pasquier also supported the existence of GBS associated with COVID-19 and presented a case from his clinic in which the patient was diagnosed with severe GBS, a type of acute inflammatory demyelinating polyneuropathy, which occurred in the wake of COVID-19 infection. Prof Du Pasquier commented on the likelihood of GBS in patients infected with SARS-CoV-2: "It is not surprising. Indeed, it is usual to see GBS after infectious viral disease."

\section{SUMMARY}

From the presented data, along with the plethora of published cases, it is evident that there are numerous neurological complications associated with SARS-CoV-2 infection. There is still speculation about the ratio between direct and
Resources like the EAN Survey on Neurological Symptoms in Patients with COVID-19 could help characterise these neurological manifestations and quantify their prevalence. What all the presenters and chairs agreed upon was perfectly encapsulated by Prof Claudio Bassetti, the EAN Acting President and chair of the first session, who gave the closing remark: "We learned a lot, but much more is to be learned."

\section{References}

1. Mao L et al. Neurologic manifestations of hospitalized patients with coronavirus disease 2019 in Wuhan, China. JAMA Neurol. 2020;77(6):683-90.

2. Harmer D et al. Quantitative mRNA expression profiling of ACE 2, a novel homologue of angiotensin converting enzyme. FEBS Lett. 2002;532(1-2):107-10.

3. Wichmann $D$ et al. Autopsy findings and venous thromboembolism in patients with COVID-19. Ann Intern Med. 2020;M20-2003. [Epub ahead of print].

4. Beyrouti R et al. Characteristics of ischaemic stroke associated with COVID-19. J Neurol Neurosurg Psychiatry. 2020;jnnp-2020-323586. [Epub ahead of print].

5. Verdoni $L$ et al. An outbreak of severe Kawasaki-like disease at the Italian epicentre of the SARS-CoV-2 epidemic: an observational cohort study. Lancet. 2020;395(10239):1771-8

6. Toscano $\mathrm{C}$ et al. Guillain-Barré syndrome associated with SARS-CoV-2. N Engl J Med. 2020;NEJMc2009191. [Epub ahead of print].

7. Zhang $T$ et al. COVID-19 associated acute disseminated encephalomyelitis - a case report. medRxiv. 2020;2020.04.16.20068148. [Preprint].

8. Poyiadji et al. COVID-19 associated acute haemorrhagic necrotising encephalopathy: CT and MRI. Available at: https://pubs.rsna.org/doi/pdf/10.1148/radiol.2020201187. Last accessed: 15 June 2020. 